\title{
Studying the small scale ISM structure with supernovae
}

\author{
F. Patat ${ }^{1}$, N. L. J. Cox ${ }^{2,3}$, J. Parrent ${ }^{4,5}$, and D. Branch ${ }^{4}$ \\ 1 European Organization for Astronomical Research in the Southern Hemisphere (ESO), K. Schwarzschild-str. 2, \\ 85748 Garching b. München, Germany \\ e-mail: fpatat@eso.org \\ 2 Herschel Science Centre, European Space Astronomy Centre, ESA, PO Box 78, 28691 Madrid, Spain \\ 3 Institute of Astronomy, K.U. Leuven, Celestijnenlaan 200D, 3001 Leuven, Belgium \\ 4 Department of Physics and Astronomy. University of Oklahoma, Norman, OK 73019, USA \\ 5 Department of Physics and Astronomy, Dartmouth College, Hanover, NH 03755-3528, Germany
}

Received 23 December 2009 / Accepted 25 February 2010

\section{ABSTRACT}

\begin{abstract}
Aims. In this work we explore the possibility of using the fast expansion of a type Ia supernova photosphere to detect extra-galactic ISM column density variations on spatial scales of $\sim 100 \mathrm{AU}$ on time scales of a few months.

Methods. We constructed a simple model which describes the expansion of the photodisk and the effects of a patchy interstellar cloud on the observed equivalent width of Na I D lines. Using this model we derived the behavior of the equivalent width as a function of time, spatial scale and amplitude of the column density fluctuations.

Results. The calculations show that isolated, small $(\leq 100 \mathrm{AU})$ clouds with Na I column densities exceeding a few $10^{11} \mathrm{~cm}^{-2}$ would be easily detected. In contrast, the effects of a more realistic, patchy ISM become measurable in a fraction of cases, and for peak-to-peak variations larger than $\sim 10^{12} \mathrm{~cm}^{-2}$ on a scale of $1000 \mathrm{AU}$.

Conclusions. The proposed technique provides a unique way to probe the extra-galactic small scale structure, which is out of reach for any of the methods used so far. The same tool can also be applied to study the sub-AU Galactic ISM structure.
\end{abstract}

Key words. supernovae: general - ISM: clouds - ISM: structure - ISM: general

\section{Introduction}

For many years it was accepted that the minimum size for the column density fluctuations in the Galactic ISM is around $1 \mathrm{pc}$ $\left(\sim 2 \times 10^{5} \mathrm{AU}\right)$. The common understanding was that although sub-parsec structures do exist, only a tiny fraction of the column density could be ascribed to these small scales (Dickey \& Lockman 1990). However, the pioneering VLBI work by Dieter et al. Romney (1976), and the later confirmation by Diamond et al. (1989) demonstrated the existence of significant fluctuations over scales of $20 \mathrm{AU}$. These findings were confirmed by $21 \mathrm{~cm}$ absorption measurements against high-velocity pulsars (Frail et al. 1991, 1994), which showed that the H I column density varies significantly over scales between 5 and $110 \mathrm{AU}$, with $10 \%-15 \%$ of the cold neutral gas distributed in AU-sized structures (Frail et al. 1994). However, some more recent radio observations on the same pulsars (Weisberg \& Stanimirović 2007) have shown that the variations are far smaller than those originally found by Frail et al. (1991, 1994).

These studies were followed by a series of works looking at the variations of $\mathrm{Ca}$ II and/or $\mathrm{Na}$ I column densities along the lines of sights to close binaries or high proper motion stars (see Crawford 2003; Lauroesch 2007, for a review). Similar investigations were carried out for molecular gas $\left(\mathrm{CH}, \mathrm{CH}^{+}\right.$, and $\mathrm{CN}$; Pan et al. 2004; Rollinde et al. 2003), and diffuse interstellar bands (Cordiner et al. 2005). An alternative method is the study of interstellar absorptions along the lines of sight to stellar clusters, like M92 (Andrews et al. 2001) and $\omega$-Cen (Van Loon et al. 2009), or the Magellanic Clouds (e.g. André et al. 2004). For a general review on the small ISM structures in our Galaxy the reader is referred to Haverkorn \& Goss (2007).

In this article we present an independent technique to analyze extra-galactic ISM structure on spatial scales of about $100 \mathrm{AU}$. The proposed method is based on the extremely high expansion velocity displayed by a supernova ( $\mathrm{SN}$ ) photosphere $\left(\sim 10^{4} \mathrm{~km} \mathrm{~s}^{-1}\right.$ or $\left.5.7 \mathrm{AU} \mathrm{day}^{-1}\right)$. A type Ia SN reaches a photospheric radius of $\sim 10^{15} \mathrm{~cm}(\sim 100 \mathrm{AU})$ in two weeks from the ex-

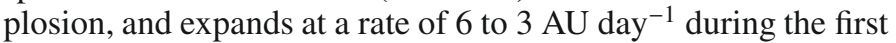
two months of its evolution. If the typical size of the fluctuations in an intervening cloud is much larger than $10^{15} \mathrm{~cm}$, then the associated absorption features will not evolve with time. On the contrary, if the ISM is patchy on comparable scales, the column density fluctuations will translate into measurable variations of the corresponding absorption features.

After introducing a simple model for the calculation of timedependent line equivalent widths for a given cloud geometry (Sect. 2), we present the results of Monte-Carlo simulations (Sect. 3), and discuss the applicability of the method and the effects on the observations of type Ia's (Sect. 4). Appendix A gives the details on the derivation of the composite equivalent width.

\section{A simple model}

Let us fix a reference polar coordinates system $(r, \theta)$ whose origin is located at the explosion center (see Fig. 1). As seen from a far observer (the distance between the SN and the observer is assumed to be much larger than that between the $\mathrm{SN}$ and the intervening material), the SN will appear as an expanding 


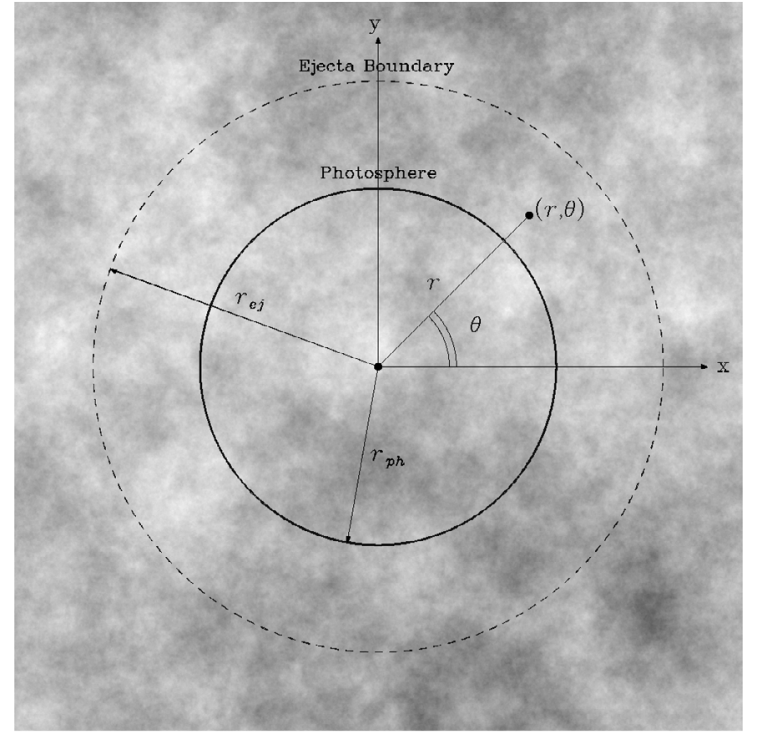

Fig. 1. Relevant quantities used in the text. The underlying column density map was generated using a power-law spectrum with $\gamma=-2.8$ (see Sect. 2.1).

photodisk which extends to the ejecta boundary radius $r_{\mathrm{ej}}(t)$. We then consider a cloud placed in front of the $\mathrm{SN}$, at a distance large enough that the explosion has no effect on its physical conditions (i.e. $>10$ pc, see Simon et al. 2009. See also Sect. 4 here), and we indicate with $N(r, \theta)$ the cloud column density in the species under consideration. Finally, we introduce $\Phi(r, t)$ as the time-dependent surface brightness profile of the photodisk at the wavelength of interest ${ }^{1}$. This function is normalized as follows

$\int_{0}^{r_{\mathrm{ej}(t)}} \int_{0}^{2 \pi} \Phi(r, t) r \mathrm{~d} \theta \mathrm{d} r=1$,

so that the total continuum flux emitted by the photodisk along the line of sight is equal to unity. If $g(N, b)$ is the curve of growth for the given transition and Doppler parameter $b$, the equivalent width $(E W)$ produced by an infinitesimal cloud column with cross section $\mathrm{d} A=r \mathrm{~d} \theta \mathrm{d} r$ is $\mathrm{d} E W=g[N, b] \Phi(r, \theta) \mathrm{d} A$.

If we neglect the small contribution by photons scattered by the cloud into the line of sight, the total equivalent width is then computed integrating the contribution of each single infinitesimal element over the photodisk (see Appendix A for the details):

$E W(t)=\int_{0}^{r_{\mathrm{ej}(\mathrm{t})}} \int_{0}^{2 \pi} g[N(r, \theta), b(r, \theta)] \Phi(r, t) r \mathrm{~d} \theta \mathrm{d} r$.

So far we have considered the possibility that the Doppler parameter $b$ can change across the cloud. However, in the lack of evidence for significant variations over the scales of interest (see for instance Welty \& Fitzpatrick 2001), in the following we will assume that $b$ is constant across the relevant portion of the cloud. We will briefly discuss the effects of a space-dependent Doppler parameter in Sect. 3.

With the aid of the outlined procedure, one can follow the time evolution of $E W$ for any input cloud column density map, provided the photodisk's expansion law is known.

In the assumption of homologous expansion (see for instance Jeffery \& Branch 1990), the radius of the photosphere $r_{\mathrm{ph}}(t)$

\footnotetext{
1 We assume $\Phi$ has no azimuthal dependence, i.e. that the $\mathrm{SN}$ is spherically symmetric. For a type Ia $\mathrm{SN}$ this is a reasonable assumption (Wang \& Wheeler 2008).
}

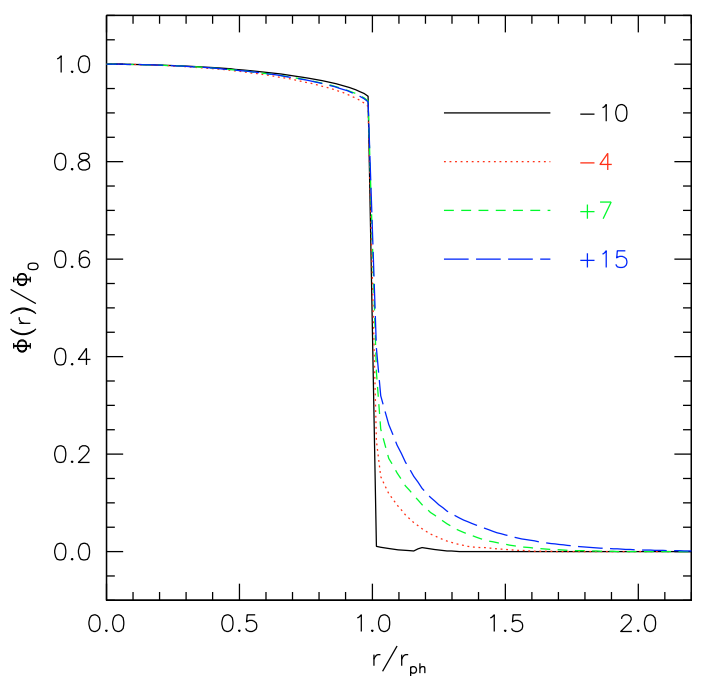

Fig. 2. Surface brightness profiles derived from SYNOW best fits of SN 1994D spectra on four different epochs $(-10,-4,+7$ and +15 days from $B$ maximum).

is obtained multiplying the photospheric velocity $v_{\mathrm{ph}}(t)$ by the time $t$ elapsed from the explosion. For $v_{\mathrm{ph}}(t)$ we adopted a best fit to values computed via SYNOW modeling of the spectroscopically normal SN 1994D, a standard type Ia event (Branch et al. 2005). During the photospheric phase $(t<100$ days $), v_{\mathrm{ph}}(t)$ is well approximated by an exponential law, and the best fit relation takes the following form:

$r_{\mathrm{ph}}(t)=\left(3.5+5.3 \mathrm{e}^{-t / 36.5}\right) t$,

where $r_{\mathrm{ph}}$ is expressed in AU and $t$ in days from the explosion. This law shows that the change in the photodisk dimensions is significant during the first two months: from day -10 to day +50 (counted from maximum light) $r_{\mathrm{ph}}$ increases by more than a factor of 5 (60 AU to $320 \mathrm{AU}$ ).

As for the photodisk surface brightness profile, this was computed using a modified version of the spectrum synthesis code SYNOW $^{2}$ (Branch et al. 2005). The profile, obtained from best fits of SN 1994D spectra for the Na I D rest-frame wavelength, rapidly drops at $r=r_{\text {ph }}$ during the early phases (see Fig. 2). As time goes by, a significant fraction of the flux (up to $\sim 16 \%$ on day +15 ) is emitted above the photosphere, and it is due to scattering by the broad Na I D doublet intrinsic to the $\mathrm{SN}$ (see for instance Jeffery \& Branch 1990). At all epochs, $\Phi=0$ for $r / r_{\mathrm{ph}} \geq 2.6$, which we used as the effective external boundary of the ejecta. To include the time dependence we tabulated $\Phi(r)$ for a number of epochs $(-10,-4,+7,+15,+28$ and +50 days from $B$ maximum) and subsequently used a linear interpolation to derive the profile at any given epoch. The time from $B$ maximum light was converted into $t$ using the rise time of SN 1994D (18 days; Vacca \& Leibundgut 1996). In view of the lack of very early spectra, we conservatively assumed that $\Phi=0$ for $r>r_{\mathrm{ph}}$ at $t=0$.

\subsection{Cloud generation}

We explored two possible cases: i) an isolated, homogeneous, spherical cloud with radius $r_{\mathrm{C}}$ and offset $x_{\mathrm{C}}$ with respect to the line of sight; ii) a patchy sheet with an input spectrum for the column density fluctuations.

\footnotetext{
${ }^{2}$ Note that SYNOW, like some other SN spectrum synthesis codes (see e.g. Mazzali \& Lucy 1993), has zero limb darkening.
} 
In the first case, the column density profile is $N\left(r^{\prime}\right)=$ $N_{0} \sqrt{1-\left(r^{\prime} / r_{\mathrm{C}}\right)^{2}}$, where $r^{\prime}$ is the projected distance from the cloud center $\left(r^{\prime} \leq r_{\mathrm{C}}\right)$, and $N_{0}$ is the column density corresponding to a ray going through the center of the cloud. The average column density is $\langle N\rangle=2 / 3 N_{0}$. As the case of an isolated, small cloud is probably quite unrealistic, it may be rather regarded as a simplified model of an over-density on an otherwise homogeneous sheet.

For the patchy sheet we adopted a procedure similar to the one described by Deshpande (2000). Molecular clouds (Elmegreen et al. 1996), the diffuse ionized component (Cordes et al. 1991), and H I (Stanimirović et al. 1999; Deshpande et al. 2000) have a fractal structure, characterized by a power-law behavior. Therefore, if $k=l_{0} / l$ is the wave-number corresponding to a given spatial scale $l$ (where $l_{0}$ is the maximum spatial scale under consideration), the power spectrum of the fluctuations can be written as $P(k) \propto k^{\gamma}$. For the large scale structure of $\mathrm{HI}$ in the SMC $\gamma \simeq-3$ (Stanimirović et al. 1999) and $\gamma=-2.75$ for the cold atomic gas in the Galaxy (Deshpande et al. 2000). Since the variations observed on scales of $\sim 100 \mathrm{AU}$ and below can be explained in terms of a single power spectrum description (Deshpande 2000), we computed the column density maps using a power-law with $\gamma=-2.8$. After generating a power spectrum map $P\left(k_{x}, k_{y}\right)$ in the Fourier plane (with $\left.k=\left(k_{x}^{2}+k_{y}^{2}\right)^{1 / 2}\right)$, we derive the real and imaginary parts of the Fourier transform of $N(x, y)$ using $\sqrt{P(k)}$ as the modulus of the complex numbers, while phases are generated as random numbers uniformly distributed between 0 and $2 \pi$. The column density map is then obtained anti-transforming into ordinary space. Given the radius of the photosphere during the time interval typically covered by observations $(<400 \mathrm{AU})$, we adopt $l_{0}=1024 \mathrm{AU}$.

Once the two-dimensional column density map is generated, it is re-normalized to have an average column density $\langle N\rangle$ and peak-to-peak fluctuations $\Delta N$ (with $\Delta N \leq 2\langle N\rangle$ ). An example cloud is presented in Fig. 1. If $\Delta N(l)$ is the column density difference between two points separated by a distance $l$, the numerical simulations show that for $\gamma=-2.8$ the RMS value of $\Delta N\left(l_{0}\right)$ is $\sim 10 \%$ of the maximum variation on the same spatial scale. Furthermore, the differences at higher wave-numbers decrease proportionally to $k^{(\gamma+2) / 2}$, as predicted by theory (Deshpande 2000). This implies that the fluctuations expected on scales of $100 \mathrm{AU}$ are $\approx 40 \%$ of those observed on scales of $1000 \mathrm{AU}$, which for Na I can reach a few $10^{12} \mathrm{~cm}^{-2}$ (Andrews et al. 2001). Therefore, a significant amount of structure is expected on spatial scales comparable to the typical photospheric radius.

\subsection{Measurement error estimates}

The ability of detecting small $E W$ variations hinges on the precision to which $E W \mathrm{~s}$ can be measured. In turn, this relates to the instrumental setup and the signal-to-noise ratio $S N R$ reached on the adjacent continuum per resolution element. To estimate the expected RMS uncertainty $\sigma_{E W}$ we have used the following formula (Chalabaev \& Maillard 1983; see their Eq. A.10), derived for photon noise-dominated conditions and with no assumption on the line profile:

$\sigma_{\mathrm{EW}}^{2}=N \frac{\delta^{2} \lambda}{S N R^{2}} \frac{F}{F_{\mathrm{c}}}+\frac{\sigma_{F_{\mathrm{c}}}^{2}}{F_{\mathrm{c}}^{2}}(\Delta \lambda-E W)^{2}$

where $\Delta \lambda$ is the line integration range, $\delta \lambda$ is the dispersion $\left(\AA \mathrm{pix}^{-1}\right), N=\Delta \lambda / \delta \lambda$ is the number of pixels corresponding to the integration range, $F$ is the average flux within the absorption,
Table 1. Estimated Equivalent Width RMS errors $\sigma_{E W}$ computed using Eq. (1) $\left(S N R=100\right.$ and $\left.N=10^{12} \mathrm{~cm}^{-2}\right)$.

\begin{tabular}{ccccccc}
\hline \hline & & \multicolumn{5}{c}{$\delta \lambda\left(\AA \mathrm{pix}^{-1}\right)$} \\
\cline { 3 - 7 } $\begin{array}{c}F W H M \\
\left(\mathrm{~km} \mathrm{~s}^{-1}\right)\end{array}$ & $b$ & 0.005 & 0.01 & 0.02 & 0.05 & 0.10 \\
& $\left(\mathrm{~km} \mathrm{~s}^{-1}\right)$ & & & & & \\
\hline \multirow{3}{*}{3.0} & 1.0 & 0.28 & 0.42 & 0.63 & 1.18 & 1.03 \\
& 3.0 & 0.35 & 0.50 & 0.74 & 1.28 & 1.83 \\
& 5.0 & 0.45 & 0.65 & 0.95 & 1.69 & 2.42 \\
\cline { 2 - 7 } 5.0 & 1.0 & 0.37 & 0.52 & 0.77 & 1.43 & 1.86 \\
& 3.0 & 0.40 & 0.57 & 0.81 & 1.53 & 2.51 \\
& 5.0 & 0.48 & 0.69 & 1.01 & 1.69 & 2.42 \\
\cline { 2 - 7 } 7.0 & 1.0 & 0.45 & 0.63 & 0.89 & 1.67 & 2.53 \\
& 3.0 & 0.46 & 0.66 & 0.93 & 1.75 & 2.51 \\
& 5.0 & 0.52 & 0.75 & 1.06 & 1.90 & 2.99 \\
\cline { 2 - 7 } 9.0 & 1.0 & 0.52 & 0.74 & 1.07 & 1.88 & 2.53 \\
& 3.0 & 0.53 & 0.76 & 1.10 & 1.75 & 3.07 \\
& 5.0 & 0.58 & 0.83 & 1.17 & 1.90 & 2.99 \\
\hline
\end{tabular}

Notes. Errors are expressed in $\mathrm{m} \AA$.

$F_{\mathrm{c}}$ is the average continuum level, and $\sigma_{F_{\mathrm{c}}}$ is the associated uncertainty. In addition to the parameters directly related to the instrumental setup, the application of this analytical expression requires the knowledge of other quantities which depend on the line profile. To compute them for a given column density, we generate synthetic Voigt profiles with input velocity parameter and a sampling equal to $\delta \lambda$. This is then convolved with a Gaussian profile with a given full width half-maximum $(F W H M)$ to mimic the instrumental broadening. Using this profile we calculate the line integration range $\Delta \lambda$, which we define as the wavelength interval in which the equivalent width is $99 \%$ of total. Within this interval we finally derive the average line flux $F$. As for the continuum $F_{\mathrm{c}}$, we assume this is computed on two symmetric, equally extended regions on either side of the absorption. If $\Delta \lambda_{\mathrm{c}}$ is the total extension of this region, then the number of pixels used to determine $F_{\mathrm{c}}$ is $N_{\mathrm{c}}=\Delta \lambda_{\mathrm{c}} / \delta \lambda$, so that $\sigma_{F_{\mathrm{c}}}^{2} / F_{\mathrm{c}}^{2}=1 /\left(N_{\mathrm{c}} S N R^{2}\right)$. In the computations we have used $\Delta \lambda_{\mathrm{c}}=2 \AA$ which, given the featureless nature of the SN pseudocontinuum, is a realistic value ${ }^{3}$.

The results obtained for $N=10^{12} \mathrm{~cm}^{-2}$, and $S N R=100$ for different values of $F W H M, b\left(1,3\right.$ and $5 \mathrm{~km} \mathrm{~s}^{-1}$ corresponding to $E W$ of 58,113 and $138 \mathrm{~m} \AA$ respectively), and $\delta \lambda$ are presented in Table 1 . As the RMS errors are inversely proportional to $S N R$, these values can be readily scaled to different signalto-noise ratios. These results have been checked against MonteCarlo simulations and were found to be consistent to within a few $0.1 \mathrm{~m} \AA$. Incidentally, this questions the need for a revision of the Chalabaev \& Maillard formula discussed by Vollmann \& Eversberg (2006).

In the following we will consider an equivalent width variation $\Delta E W$ detectable if $|\Delta E W| \geq 5 \sqrt{2} \sigma_{E W}$. For a typical case where $F W H M=7 \mathrm{~km} \mathrm{~s}^{-1}, b=1 \mathrm{~km} \mathrm{~s}^{-1}, \delta \lambda=0.01 \AA$ $\mathrm{pix}^{-1}$, and $S N R=100$, this turns into a 5- $\sigma$ detection limit $\Delta E W_{\text {lim }}=4.4 \mathrm{~m} \AA\left(\Delta E W_{\lim }=5.3 \mathrm{~m} \AA\right.$ for $\left.b=5 \mathrm{~km} \mathrm{~s}^{-1}\right)$.

\footnotetext{
3 For the purposes of measuring the $E W$ of an inter-stellar line, the continuum definition in a SN spectrum is much easier than in a stellar spectrum, where the presence of other intrinsic features may contaminate the adjacent regions.
} 


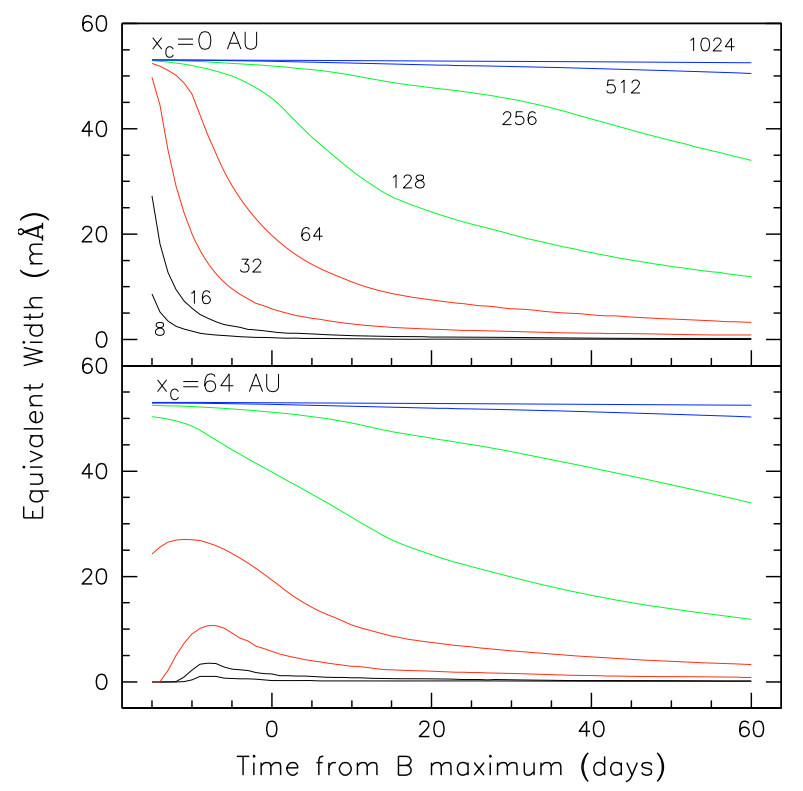

Fig. 3. Examples of simulated $\mathrm{Na} I \mathrm{D}_{2} E W$ variation as a function of time for a spherical, homogeneous cloud with offset $x_{\mathrm{C}}=0$ (upper panel) and $x_{\mathrm{C}}=64 \mathrm{AU}$ ((lower panel). The various curves refer to different cloud radii $r_{\mathrm{C}}(8,16,32,64,128,256,512$ and $1024 \mathrm{AU}$ from bottom to top). In all cases $N_{0}(\mathrm{NaI})=5 \times 10^{11} \mathrm{~cm}^{-2}$ and $b=1 \mathrm{~km} \mathrm{~s}^{-1}$.

\section{Results of simulations}

Although the model can be used for any inter-stellar absorption line, in the following we present the results obtained for $\mathrm{NaI} \mathrm{D}_{2}$, because it is a strong transition, it falls in a region almost free of telluric absorption features, and in a spectral interval where most optical, high-resolution spectrographs have their maximum sensitivity.

\subsection{Isolated spherical cloud}

Example $E W$ evolutions for two different cloud offsets $x_{\mathrm{C}}(0$ and $64 \mathrm{AU})$ and a number of cloud radii $r_{\mathrm{C}}$ are presented in Fig. 3 up to 2 months after maximum light. In general, the maximum variability is expected when the cloud is close to the center of the photodisk. The maximum variation is achieved for cloud radii between 64 and 128 AU. Also, small clouds are better detected during the early phases (when their size is comparable to that of the photosphere), while the detection of large clouds requires a larger time span. If the cloud is too large $\left(r_{\mathrm{C}}>512 \mathrm{AU}\right)$, then the $E W$ variation is not sufficiently ample to be detected. With a minimum set of two observations taken 10 days apart in the pre-maximum phases, for typical high-resolution setup, a 5- $\sigma$ detection limit of $4.4 \mathrm{m \AA}, b=1 \mathrm{~km} \mathrm{~s}^{-1}$ and $N_{0}=5 \times 10^{11} \mathrm{~cm}^{-2}$, the simulations show that one is able to detect clouds with $r_{\mathrm{C}}$ between 16 and 128 AU up to a maximum offset of 64 AU. For larger offsets the cloud starts to intersect the photodisk when its size is too large and the corresponding covering factor is too small. Besides implying probably unrealistic density contrasts, increasing the column density does not enhance the detectability of a small size cloud, since this rapidly becomes totally opaque. We note that, while this causes the saturation of the covering factor, it does not produce a saturated profile in the emerging absorption line.

A distinctive feature of small $\left(r_{\mathrm{C}} \leq 64 \mathrm{AU}\right)$, offset clouds is an $E W$ growth followed by a decrease during the premaximum light phase (Fig. 3, lower panel). This is due to the growth of the covering factor as the photodisk starts intersecting the off-centered knot. Once a maximum value is reached, the subsequent increase in the photodisk size causes the covering factor to drop. Although this mechanism can produce an absorption feature which grows in strength and then disappears on timescales of a month, this is expected to happen only during the pre-maximum epochs, when the relative increase in the photodisk surface is very fast. Larger clouds placed at larger offsets $\left(x_{\mathrm{C}} \geq 256 \mathrm{AU}\right)$ produce features which start appearing around maximum light, but keep steadily increasing in strength up to several months after maximum.

Finally, for central column densities (or density contrasts) smaller than $\sim 2 \times 10^{10} \mathrm{~cm}^{-2}$, the $E W$ variations are always below the detection limit.

\subsection{Patchy clouds}

Because of the stochastic geometry of the power-law clouds, their effects were evaluated using a statistical approach. For a given cloud realization we computed $E W(t)$ and derived the absolute peak-to-peak variation $\triangle E W$ over the whole time interval -10 to +50 days. To mimic a more realistic situation, we did this also on two sub-sets of data-points including only postmaximum observations $(0,+10,+20,+30,+40,+50$ and $0,+10$, $+20,+30$ days). As statistical estimators we computed the median absolute peak-to-peak variation over the full range $(\overline{\Delta E W})$, the semi-inter-quartile range and the 99-th percentile $\left(\Delta E W_{99}\right)$. For the three time ranges we finally estimated the 5- $\sigma$ detection probabilities $P_{t}(-10 \leq t \leq+50), P_{0}(t \geq 0)$ and $P_{30}$ $(0 \leq t \leq+30)$. The results are presented in Table 2 for different values of $\langle N\rangle, \Delta N$ and $b$. For each parameter set 5000 cloud realizations were computed (blank values indicate detection probabilities $\left\langle 2 \times 10^{-4}\right)$. $\langle E W\rangle$ indicates the $E W$ corresponding to $\langle N\rangle$ and the input Doppler parameter.

Visual inspection of a number of realizations showed that the $E W(t)$ curves are smooth, with typical timescales of the order of 10 days. The variation rate is systematically larger at early epochs and decreases as time goes by. The simulations show that peak-to-peak column density fluctuations smaller than $10^{11} \mathrm{~cm}^{-2}$ on a scale of $\sim 1000$ AU do not produce any measurable effects. Even with high $(\sim 100)$ signal-to-noise ratio observations, starting 10 days before maximum and covering the first two months of the SN evolution, the detection probability is below $2 \%$. This grows significantly when the fluctuations exceed $\sim 10^{12} \mathrm{~cm}^{-2}$. Incidentally, this implies that SNe suffering higher extinction are expected to display more pronounced variations (see also Chugai 2008). Because of the saturation effect, the $E W$ variations are more marked for larger values of the Doppler parameter. Finally, for column densities exceeding $\sim 10^{13} \mathrm{~cm}^{-2}$ the detection probability decreases due to line saturation.

To study the effect of a spatially variable Doppler parameter, we have run a set of simulations in which $b$ is allowed to fluctuate around the average value across the cloud. We have tentatively modeled the Doppler parameter map using the same algorithm and spatial scales spectrum adopted for the column density generation (see Sect. 2.1). We remark that this is not meant to reproduce real physical conditions, but only to estimate the consequences of velocity dispersion fluctuations (for instance, $b$ and $\langle N\rangle$ were left completely independent). The MC runs (with $1 \leq b \leq 5 \mathrm{~km} \mathrm{~s}^{-1}$ ) show that for $\langle N\rangle<10^{11} \mathrm{~cm}^{-2}$ there is practically no difference with respect to the case with constant Doppler parameter. The differences start to be significant at $\langle N\rangle>10^{12} \mathrm{~cm}^{-2}$, for which the typical variations and detection probabilities become much larger. 
Table 2. Results of Monte-Carlo simulations for power-law clouds with $\gamma=-2.8$.

\begin{tabular}{cccccccc}
\hline \hline \multicolumn{7}{c}{$\gamma=-2.8, b=1.0 \mathrm{~km} \mathrm{~s}^{-1}, \Delta E W_{\lim }=4.4 \mathrm{m \AA}$} \\
\hline$\langle N\rangle$ & $\Delta N$ & $\langle E W\rangle$ & $\overline{\Delta E W}$ & $\Delta E W_{99}$ & $P_{t}$ & $P_{0}$ & $P_{30}$ \\
$\left(10^{12} \mathrm{~cm}^{-2}\right)$ & $\mathrm{m} \AA$ & $\mathrm{m} \AA$ & $\mathrm{m} \AA$ & $\%$ & $\%$ & $\%$ \\
\hline 0.1 & 0.1 & 16.3 & $0.6(0.3)$ & 2.2 & - & - & - \\
0.1 & 0.2 & & $1.3(0.6)$ & 4.5 & 1.5 & - & - \\
1.0 & 1.0 & 57.7 & $0.7(0.4)$ & 2.7 & - & - & - \\
1.0 & 2.0 & & $1.5(0.8)$ & 8.0 & 5.0 & 2.1 & 1.1 \\
10.0 & 10.0 & 85.7 & $0.6(0.3)$ & 1.9 & - & - & - \\
10.0 & 20.0 & & $1.1(0.5)$ & 5.1 & 1.8 & 0.6 & 0.2 \\
\hline \multicolumn{7}{c}{$\gamma=-2.8, b=5.0 \mathrm{~km} \mathrm{~s}^{-1}, \Delta E W_{\lim }=5.2 \mathrm{m \AA}$} & \\
\hline 0.1 & 0.1 & 18.9 & $0.9(0.4)$ & 3.1 & - & - & - \\
0.1 & 0.2 & & $1.7(0.8)$ & 6.2 & 2.3 & 0.1 & - \\
1.0 & 1.0 & 138.0 & $4.6(2.1)$ & 15.6 & 40.7 & 22.2 & 9.8 \\
1.0 & 2.0 & & $9.1(4.3)$ & 33.9 & 77.1 & 56.1 & 40.3 \\
10.0 & 10.0 & 334.0 & $3.1(1.4)$ & 11.1 & 19.2 & 7.1 & 2.4 \\
10.0 & 20.0 & & $6.3(3.1)$ & 32.6 & 60.3 & 40.6 & 25.2 \\
\hline \multicolumn{7}{c}{}
\end{tabular}

Notes. Equivalent width variations are peak-to-peak (see Sect. 3.2). Values in parenthesis indicate the semi-inter-quartile range.

This was to be expected, since in the quasi-linear regime attained at low column densities the effect tends to average out. On the contrary, as one enters the non-linear part of the curve of growth, the largest variations are produced by the regions of the cloud where the Doppler parameter is higher (i.e. less subject to saturation), thus skewing the distribution towards more marked $E W$ fluctuations. The exact behavior depends on the way the velocity dispersion varies across the cloud and how this (if any) relates to the column density fluctuations. However, the conclusion that a variable Doppler parameter enhances the detection probability is of general validity.

\section{Discussion and conclusions}

The simulations presented in this paper indicate that marked time effects on the measured $E W \mathrm{~s}$ are expected for small $\left(r_{\mathrm{C}} \leq\right.$ $100 \mathrm{AU})$, isolated clouds with $N_{0}(\mathrm{NaI}) \sim 10^{11}-10^{12} \mathrm{~cm}^{-2}$ and for small offsets $\left(x_{\mathrm{C}}<100 \mathrm{AU}\right)$. However, the existence of such structures is seriously questioned in terms of pressure equilibrium arguments and the yet unknown processes that would produce them (see Heiles 1997, and references therein). Frail et al. (1994) detected maximum $\Delta N(\mathrm{H} \mathrm{I})$ variations that range from $\sim 10^{19}$ to $\sim 5 \times 10^{20} \mathrm{~cm}^{-2}$ on scales between 5 and $100 \mathrm{AU}$. For a Galactic Na I/H I ratio (Ferlet et al. 1985) this turns into $\Delta N\left(\mathrm{Na}\right.$ ) between $5 \times 10^{10}$ and $3 \times 10^{12} \mathrm{~cm}^{-2}$. These large changes have been interpreted as arising within ubiquitously distributed small structures. However, this picture has been questioned by Deshpande (2000), who has convincingly shown that the observations are consistent with a single power-law description of the ISM, down to AU scales. In these circumstances, peak-to-peak variations $\Delta N(\mathrm{NaI}) \sim 10^{12} \mathrm{~cm}^{-2}$ are expected on scales of $\sim 100$ AU. Our calculations (see Table 2) show that for a type Ia SN observed under the most favorable conditions ( $S N R>100$ on all epochs, spanning from -10 to +50 days) these would appear in less than $10 \%$ of the cases for $b \sim 1 \mathrm{~km} \mathrm{~s}^{-1}$. This fraction increases to $\sim 80 \%$ for $b \sim 5 \mathrm{~km} \mathrm{~s}^{-1}$, but in all cases it is $\Delta E W \leq 40 \mathrm{~m} \AA$. These small variations imply negligible changes in $E(B-V)$, but can have some effect on observing programmes studying the evolution of $\mathrm{Na}$ I features possibly arising in the circumstellar environment of type Ia progenitors (Patat et al. 2007; Simon et al. 2009). However, we note that these values are more than a factor of 10 smaller than the $\mathrm{Na}$ I $\mathrm{D}$ variations detected in the type Ia SN 2006X (Patat et al. 2007), which were attributed to the ionization effects induced by the $\mathrm{SN}$ on its circum-stellar environment. In contrast, no statistically significant variations were detected for the $\mathrm{CN}, \mathrm{CH}, \mathrm{CH}^{+}$, $\mathrm{Ca}$ I lines and DIBs associated to an interstellar cloud in the host galaxy (Patat et al. 2007; Cox \& Patat 2008). In the only other well studied case published so far (SN 2007le), the $E W$ of four Na I D components remained constant to within a few $\mathrm{m} \AA$ during six epochs spanning about 3 months (Simon et al. 2009). In this time range $r_{\mathrm{ph}}$ changed approximately from 100 to $400 \mathrm{AU}$, and the lack of evolution is in line with the predictions of our model for a power spectrum ISM and definitely excludes the presence of small, isolated clouds with sizes comparable to $r_{\mathrm{ph}}$. Although the available data are still scanty, the multi-epoch, high-resolution campaigns which are being conducted for the study of type Ia progenitors will provide a more statistically significant sample.

All the discussion so far is based on the assumption that the physical conditions of the ISM are not modified by the SN explosion, so that all variations in the absorption lines are due to pure geometric effects. Indeed, a type Ia SN can produce changes in the ionization balance of low-ionization species (like $\mathrm{Na}$ I or $\mathrm{K}$ I) up to quite large distances, of the order of 10 pc (Patat et al. 2007; Chugai 2008; Simon et al. 2009). Given the UV flux predicted for these distances (Simon et al. 2009), the ionization timescale of $\mathrm{Na}$ I is expected to be about 120 days. Since the electron density in the ISM is low, the recombination time is extremely long. Therefore, under the assumption of a constant ionizing field, one would expect the amount of neutral $\mathrm{Na}$ to decrease with timescales of several months, hence mimiking small scale structure effects. However, the UV flux of a type Ia SN decreases significantly after maximum light (a factor $\sim 20$ in the first 40 days; Brown et al. 2009) implying that the maximum distance is probably less than $10 \mathrm{pc}$. Given the range of possible distances to an inter-stellar cloud within the host galaxy, this suggests that significant $\mathrm{Na}$ I column density variations in the ISM induced by the $\mathrm{SN}$ radiation field are improbable.

Another important fact is that, because of the much higher ionization potential of $\mathrm{Ca}$ II, and the strong UV line blocking present in type Ia spectra, the $E W$ of the ubiquitous $H \& K$ lines becomes insensitive to the $\mathrm{SN}$ radiation field already at distances of a few $0.1 \mathrm{pc}$ (Simon et al. 2009). In contrast, in the case of a geometrical origin, all species are expected to show synchronous variations, although possibly with different amplitudes and time scales (Lauroesch \& Meyer 2003). Therefore, a comparison between the behaviors of $\mathrm{Na}$ I and $\mathrm{Ca}$ II should allow one to disentangle between geometrical and ionization effects, similar to what has been proposed by Patat et al. (2007).

The method we presented enables the study of small scale structure in the extragalactic ISM, which is out of reach for any of the techniques deployed so far. In this respect we note that the same method can be in principle applied to the Galactic ISM to study its sub-AU structure, for which no direct measurements are available yet. Although probably requiring very high signalto-noise ratios, this technique might put important constraints on the very small scale structure. In this article we have discussed the case of the strong, easily detectable Na I D lines. However, other weaker lines (e.g., K I, Ca I, Ca II) can be used, especially when the $\mathrm{Na}$ I D lines are saturated. In general, the simultaneous study of different atomic/diatomic lines along the lines of sight to SNe will contribute to get a more detailed picture of the physical conditions of the ISM in the small scales regime. 


\section{Appendix A: Calculation of composite equivalent width}

Let us consider an extended photodisk, a cloud placed in front of it and an absorption line with profile function $\phi(v)$, normalized so that

$\int_{0}^{+\infty} \phi(v) \mathrm{d} v=1$.

If $N$ is the space-dependent column density of the cloud (atoms $\mathrm{cm}^{-2}$ ) for the atomic species under consideration, the monochromatic line optical depth is $\tau(v)=K N \phi(v)$, where $K$ is the frequency-integrated line opacity $\left(\mathrm{cm}^{2}\right.$ atom $\left.{ }^{-1}\right)$. Let us now consider an infinitesimal element of the photodisk $\mathrm{d} A=r \mathrm{~d} \theta \mathrm{d} r$ in the polar reference system introduced in Sect. 2 (see Fig. 1), and define $\Phi(r)$ as the radial profile of continuum surface brightness for the photodisk. For the sake of simplicity we assume that the continuum $I_{0}$ is constant across the line profile and is normalized to unity:

$I_{0}=2 \pi \int_{0}^{r_{\mathrm{ej}}} \Phi(r) r \mathrm{~d} \theta \mathrm{d} r=1$.

With these settings, the monochromatic intensity contributed by the infinitesimal photodisk element $\mathrm{d} A$ to the total observed intensity can be written as

$d I(v, r, \theta)=\mathrm{e}^{-K N(r, \theta) \phi(v)} \Phi(r) r \mathrm{~d} \theta \mathrm{d} r$.

For a distant observer, to whom the photodisk will appear as an unresolved source, the total line intensity profile $I(v)$ is obtained integrating all the infinitesimal contributions over the photodisk:

$I(v)=\int_{0}^{r_{\mathrm{ej}}} \int_{0}^{2 \pi} \mathrm{e}^{-K N(r, \theta) \phi(v)} \Phi(r) r \mathrm{~d} \theta \mathrm{d} r$.

Given the definition of equivalent width

$E W=\int_{0}^{+\infty}\left(1-\frac{I(v)}{I_{0}}\right) \mathrm{d} v$

the composite equivalent width can be expressed as follows:

$E W=\int_{0}^{+\infty}\left(1-\int_{0}^{r_{\mathrm{ej}}} \int_{0}^{2 \pi} \mathrm{e}^{-K N(r, \theta) \phi(v)} \Phi(r) r \mathrm{~d} \theta \mathrm{d} r\right) \mathrm{d} \nu$.

Because of the normalization of $\Phi(r)$, the previous relation can be rewritten as

$E W=\int_{0}^{r_{\mathrm{ej}}} \int_{0}^{2 \pi} \int_{0}^{+\infty}\left(1-\mathrm{e}^{-K N(r, \theta) \phi(v)}\right) \Phi(r, \theta) r \mathrm{~d} \theta \mathrm{d} r \mathrm{~d} v$

Now, the inner integral is the equivalent width one would observe if the physical system were composed only by the infinitesimal cloud element $\mathrm{d} A$ and the corresponding portion of the photodisk:

$E W(r, \theta)=\int_{0}^{+\infty}\left(1-\mathrm{e}^{-K N(r, \theta) \phi(v)}\right) \mathrm{d} \nu$ which implies that the composite equivalent width is the weighted sum of the equivalent widths produced within each infinitesimal cloud element. If $g(N, b)$ is the curve of growth for the given transition (where $b$ is the Doppler parameter that characterizes the line profile), then Eq. (A.1) can be reformulated as:

$E W=\int_{0}^{r_{\mathrm{ej}}} \int_{0}^{2 \pi} g[N(r, \theta), b(r, \theta)] \Phi(r, \theta) r \mathrm{~d} \theta \mathrm{d} r$.

Acknowledgements. The authors wish to thank L. Tacconi-Garman, S. Stanimirović and A. Deshpande for their kind help. The authors are also grateful to an anonymous referee for the useful comments and suggestions.

\section{References}

André, M. K., Le Petit, F., Sonnentrucker, P., et al. 2004, A\&A, 422, 483 Andrews, S. M., Meyer, D. M., \& Lauroesch, J. T. 2001, ApJ, 552, L73 Branch, D., Baron, E., Hall, N., et al. 2005, PASP, 117, 545 Brown, P. J., Holland, S. T., Immler, S., et al. 2009, ApJ, 137, 4517

Chalabaev, A., \& Maillard, J. P. 1983, A\&A, 127, 279

Chugai, N. N. 2008, Astron. Lett., 34, 389

Cordes, J. M., Weisberg, J. M., Frail, D. A., Spangler, S. R., \& Ryan, M. 1991, Nature, 354, 121

Cordiner, M., Sarre, P., \& Fossey, S. 2005, AAO News Letter, 107, 9

Cox, N. L.J., \& Patat, F. 2008, A\&A, 485, L9

Crawford, I. A., Howarth, I. D., Ryder, S. D., \& Stathakis, R. A. 2000, MNRAS, 319. L1

Crawford, I. A. 2003, Ap\&SS, 285, 661

Deshpande, A. A. 2000, MNRAS, 317, 199

Deshpande, A. A., Dwarakanath, K. S., \& Goss, W. M. 2000, ApJ, 543, 227

Dickey, J. M., \& Lockman, F. J. 1990, ARA\&A, 28, 215

Diamond, P. J., Goss, W. M., Romney, J. D., et al. 1989, ApJ, 347, 302

Dieter, N. H., Welch, W. J., \& Romney, J. D. 1976, ApJ, 206, L113

Elmegreen, B. G., \& Falgarone, E. 1996, ApJ, 471, 816

Ferlet, R., Vidal-Madjar, A., \& Gry, C. 1985, ApJ, 298, 838

Frail, D. A., Cordes, J. M., Hankins, T. H., \& Weisberg, J. M. 1991, ApJ, 382, 168

Frail, D. A., Weisberg, J. M., Cordes, J. M., \& Mathers, A. 1994, ApJ, 436, 144

Haverkorn, M., \& Goss, W. M. 2007, SINS - Small Ionized and Neutral Structures in the Diffuse Interstellar Medium, ed. Haverkorn, M., \& Goss, W. M. (San Francisco: ASP), ASP Conf. Ser., 365

Heiles, C. 1997, ApJ, 481, 193

Jeffery, D., \& Branch, D. 1990, Jerusalem Winter School for Theoretical Physics, Supernovae, ed. J. C. Wheeler, T. Piran, \& S. Weinberg (Singapore: World Scientific Publishing Co.), 149

Lauroesch, J. T., \& Meyer, D. M. 2003, ApJ, 591, L123

Lauroesch, J. T. 2007, in SINS - Small Ionized and Neutral Structures in the Diffuse Interstellar Medium, ed. M. Haverkorn, \& W. M. Goss (San Francisco: ASP), ASP Conf. Ser., 365, 40

Mazzali, P. A., \& Lucy, L. B. 1993, A\&A, 279, 447

Pan, K., Federman, S. R., Cunha, K., Smith, V. V., \& Welty, D. E. 2004, ApJS, 151,313

Patat, F., Chandra, P., Chevalier, R., et al. 2007, Science, 317, 924

Rollinde, E., Boissé, P., Federman, S. R., \& Pan, K. 2003, A\&A, 401, 215

Simon, J. D., Gal-Yam, A., Gnat, O., et al. 2009 ApJ, 702, 1157

Stanimirović, S., Stavely-Smith, S. L., Dickey, J. M., Sault, R. J., \& Snowden, S. L. 1999, MNRAS, 302, 417

Vacca, W. D., \& Leibundgut, B. 1996, ApJ, 471, L37

Van Loon, J. Th., Smith, K. T., McDonald, I., et al. 2009, MNRAS, 399, 195

Vollmann, K., \& Eversberg, T. 2006, Astron. Nachr., 327, No. 9, 862

Wang, L., \& Wheeler, J. C. 2008, ARA\&A, 46, 433

Weisberg, J. M., \& Stanimirović, S. 2007, in SINS - Small Ionized and Neutral Structures in the Diffuse Interstellar Medium, ed. M. Haverkorn, \& W. M. Goss (San Francisco: ASP), ASP Conf. Ser., 365, 28

Welty, D. E., \& Fitzpatrick, E. L. 2001, ApJ, 551, L175 